\title{
Síndrome de úlceras gástricas en equinos
}

Patricio Razquin-Echeverriarza ${ }^{1} \bowtie$, Mauricio Ureña-Durán, José Vargas-Arrieta ${ }^{1}$, Roberto Estrada-

McDermott ${ }^{1}$, Juan Estrada-Umaña ${ }^{1}$

1 Hospital de equinos, Universidad Nacional. Email: patorazquin@gmail.com, josevargas29@gmail.com, roberto.estrada.modermott@una.cr

Las úlceras gástricas son uno de los padecimientos más comunes en los equinos, con una prevalencia que varía desde un $10 \%$ hasta un $95 \%$ según estudios realizados en diferentes países del mundo. Actualmente en Costa Rica no existen investigaciones científicas sobre la patología, sin embargo, esta forma parte de los diagnósticos diferenciales en casos de pérdida de peso crónica, bruxismo y halitosis. Si bien los signos clínicos son sugestivos de la enfermedad, se requiere confirmar el diagnóstico por medio de una gastroscopia, la cual permite observar de forma directa las úlceras. Para poder realizar este procedimiento correctamente no solo es necesario el equipo adecuado, sino que también se requiere conocimiento de la anatomía y fisiopatología de la enfermedad para optimizar los resultados del tratamiento.

\section{Objetivos}

Informar a los médicos veterinarios y estudiantes de la carrera de Medicina Veterinaria de Costa Rica sobre la importancia de esta patología, así como su fisiopatología y las bases anatómicas necesarias para poder diagnosticarla correctamente y tratarla, además de poder educar a los propietarios sobre las medidas preventivas para dicha enfermedad.

\section{Resultados}

A lo largo de los años se han realizado múltiples procedimientos de gastroscopia en el Hospital de Especies Mayores de la Universidad Nacional, con los que se ha logrado diagnosticar y tratar el síndrome de úlcera gástrica.

\section{Conclusiones}

El síndrome de úlceras gástricas en equinos es una enfermedad frecuente en la medicina equina, que se puede prevenir de forma satisfactoria al fomentar medidas de manejo adecuadas, las cuales deben ser recomendadas por el médico veterinario, por lo que este debe conocer la patología y sus implicaciones. Sin embargo, cuando la enfermedad se ha desarrollado, es importante realizar un diagnóstico preciso, el cual se logra por medio de la gastroscopia. Una vez realizado el diagnóstico, el pronóstico suele ser bueno siempre y cuando se aplique un tratamiento adecuado junto con las medidas preventivas necesarias.

Autor para correspondencia Patricio Razquin-Echeverriarza: patorazquin@gmail.com 